\title{
Large Eddy Simulation Applied to Reciprocating Compressors
}

\author{
César J. Deschamps \\ Senior Member, ABCM \\ deschamps@polo.ufsc.br \\ Departamento de Engenharia Mecânica \\ Universidade Federal de Santa Catarina - UFSC \\ 88040-900 Florianópolis, SC, Brazil
}

This paper considers the application of large-eddy simulation (LES) to predict the performance of hermetic reciprocating compressors utilized in refrigeration. In such devices the pressure difference between the suction/discharge chamber and the cylinder, established by the piston motion, is responsible for the valve opening. Once the valves are open, the flow dictates the pressure distribution on the valve reed surface and, consequently, the resultant force that will govern the valve dynamics and its displacement from the seat. The methodology developed herein applies LES, combined with the Smagorinsky sub-grid model, to account for the compressible turbulent flow through the discharge valve. A one-degree of freedom model is adopted for the valve dynamics, and a finite volume methodology to solve the flow field throughout the discharge valve. For the remainder of the compression cycle, an integral formulation is employed, with effective flow and force areas being used to evaluate the dynamics and mass flow rate for the suction valve. Numerical results demonstrate that the methodology is capable of predicting important flow features in the discharge process, at a reasonable computational cost. Keywords: Automatic valves, reed valves, reciprocating compressor simulation

\section{Introduction}

The role of the compressor in a refrigeration system is to establish the pressure difference between the condensing and evaporating lines and also to supply the required mass flow rate of refrigerant. Compressors can be divided into two groups: rotodynamic (centrifugal, axial, radial, etc) and positive displacement (reciprocating, rotary, scroll, screw, etc). A roto-dynamic machine subjects a steadily flow of gas to forces that result in a continuous rise in pressure. Positive displacement compressors take in an amount of gas and trap it inside a volume that is diminished by deformation. In this process, the pressure rises and when it reaches a certain value the gas is pushed out against the pressure in the discharge chamber. Hence, in a positive displacement compressor the flow is not steady, occurring rather in pulses.

Figure 1 presents a schematic view of a reciprocating compressor and the indicator diagram for a typical cycle. When the piston moves downwards, it reaches a position where low pressure vapor is drawn in through the suction valve, which is opened automatically by the pressure difference between the cylinder and the suction chamber. The vapor keeps flowing in during the suction stroke as the piston moves towards the bottom dead center (BDC), filling the cylinder volume with vapor at suction pressure, $\mathrm{p}_{\mathrm{s}}$. The suction process is represented by curve $\mathrm{A}-\mathrm{C}$ in the indicator diagram of Fig. 1b. After reaching the BDC, the piston starts to move in the opposite direction, the suction valve is closed, the vapor is trapped, and its pressure rises as the cylinder volume decreases. Eventually, the pressure reaches the pressure in the discharge chamber, $\mathrm{p}_{\mathrm{d}}$, and the discharge valve is forced to open. After the opening of the discharge valve, the piston keeps moving towards the top dead center (TDC), represented by point A. It should be noted that suction and discharge processes do not take place at constant pressure. This phenomenon is associated with the dynamics of the valves and the restriction imposed by the valve passage areas. This appears on the indicator diagram, with compression continuing after pressure $p_{d}$ is reached and the same happening for the expansion stroke after pressure $\mathrm{p}_{\mathrm{s}}$.

At point $\mathrm{A}$ there remains an amount of refrigerant in the cylinder at discharge pressure, since a gap between the piston and cylinder head is necessary to accommodate the valves and also to

Presented at ETT $2004-4^{\text {th }}$ Spring School on Transition and Turbulence September $27^{\text {th }}$ - October $1^{\text {st }}, 2004$, Porto Alegre, RS, Brazil.

Paper accepted: May, 2005. Technical Editor: Aristeu da Silveira Neto. allow for manufacturing tolerances. The volume resulting from such a clearance may account for up to $5 \%$ of the total cylinder volume. Therefore, as the piston moves downwards, the refrigerant in the clearance is re-expanded and its pressure decreases, as represented by process $\mathrm{AB}$. When the piston reaches point $\mathrm{B}$, the cylinder pressure is lower than the suction chamber pressure, $\mathrm{p}_{\mathrm{s}}$, causing the suction valve to open and the gas to flow into the cylinder up to approximately point $\mathrm{C}$. Hence, instead of taking in a volume of vapor equal to the volume $\left(\mathrm{V}_{\mathrm{C}^{-}}-\mathrm{V}_{\mathrm{A}}\right)$, a smaller volume $\left(\mathrm{V}_{\mathrm{C}}-\mathrm{V}_{\mathrm{B}}\right)$ is drawn from the suction chamber, reducing the compressor volumetric efficiency. Other aspects also affect the compressor efficiency, such as heat transfer between the refrigerant and the cylinder wall, throttling in valve ports, gas leakage through the clearance between the piston and the bore, backflow in the valves, etc.

Most domestic refrigeration systems adopt hermetic reciprocating compressors, due to a number of features: small volume, low levels of noise and energy consumption, absence of leakage, among others. In hermetic models, motor and compressor are directly coupled on the same shaft and the assembly is installed inside a welded steel shell. An important disadvantage of hermetic compressors is that it is not possible to service them without cutting the shell. Therefore, the compressor must be manufactured in such a way that it can last the life of the system.

Reed valves are essential parts in hermetic compressors. These valves are called automatic because they open and close depending on the pressure difference between the cylinder and the suction/discharge chamber, established by the piston motion. Once the valves are open, the pressure flow field is responsible for the resultant force acting on the reed. In designing the valve system for reciprocating compressors four main features related to the valve performance are sought: fast response, high mass flow rate, low pressure drop when opened, and good backflow blockage when closed. For this reason, and in order to obtain an optimum valve system, it is crucial to recognize and predict the phenomena associated with the flow through the valve as well as to its dynamics.

Despite their geometric simplicity, automatic valves possess a complex mathematical description, usually requiring some simplifying hypotheses. For this reason, several studies available in the literature related to automatic valves either model the valve dynamics in detail but pay little attention to the description of the flow field (Khalifa and Liu, 1998), or focus on the fluid mechanics without considering the coupling between valve motion and pressure distribution on the reed (Deschamps et al., 1996). Trella 
and Soedel (1974) developed a methodology to analyze the nonlinear behavior of discharge valve systems, considering a transient compressible flow. The valve dynamics was coupled to the flow field, which was solved from integral conservation laws written for a number of control volumes for the flow through the valve. The study demonstrated the importance of solving the valve dynamics coupled with the flow field of the valve. More recently, Lopes and Prata (1997) developed a numerical methodology to explore the interaction between valve dynamics and fluid flow. By prescribing a periodic velocity profile at the entrance of the valve orifice, and solving the continuity and momentum equations via the finite volume technique, the flow field was obtained. From the pressure on the reed the resultant force was determined and a one-dimensional dynamic model was employed to solve the reed acceleration, velocity and displacement. Later, Matos et al. (2002) extended the model of Lopes and Prata (1997) so as to take into account the turbulent regime that prevail in valves.

In the context of compressor simulation, a number of important studies can be cited. Soedel (1972) presents a model to thermodynamically evaluate the performance of compressors under cyclical conditions, using integral relations for conservation of mass and energy, and a dynamic model for valves. MacLaren et al. (1975) adopted conservation equations in differential form and solved the unsteady one-dimensional compressible fluid flow through suction and discharge systems with the method of characteristics. PérezSegarra et al. (1994) proposed a numerical procedure, based on the finite volume method, to simulate the transient compressible onedimensional flow in the compressor. The most elaborate model put forward so far is that of Matos et al. (2002), where a twodimensional model was used to simulate the complete compression cycle, including the turbulent flow through the discharge valve. Turbulence contribution to the flow transport was accounted for with the RNG k- $\varepsilon$ model. The methodology was found to return a precise description of physical mechanisms that influence the compressor efficiency, such as heat transfer at the cylinder wall and transient effects on the valve dynamics. Despite such promising predictions, Matos et al. (2002) pointed out that computational processing time was excessive for compressor design purposes.

The main objective of this work is to offer an alternative to the compressor simulation methodology of Matos et al. (2002), with the same level of accuracy but with a much lower computational cost.

\section{Mathematical Model}

The necessary reduction in the computational cost is sought here through two approaches. Firstly, the simulation methodology combines differential and integral formulations for the governing equations. Differential equations are solved to capture flow details during the opening of the discharge valve, which are crucial to correctly predict its dynamics. The flow through the suction valve could be solved in the same way but, following Matos et al. (2002), this treatment was applied to the discharge valve only, at this stage. Therefore, for the remainder of the compressor cycle, including the opening of the suction valve, an integral formulation is used. The other strategy is to replace the RNG k- $\varepsilon$ turbulence model adopted in Matos et al. (2002) with large-eddy simulation (LES). The number of differential equations in LES is smaller and, given that flow in compressors is naturally transient, this may be effective in reducing the computational effort. More details on both formulations are given next.

\section{Integral Formulation}

The integral formulation adopted to simulate the compressor is a simplified version of the code proposed by Ussyk (1984). The code accounts for piston displacement as a function of crankshaft angle, the thermodynamic process inside the cylinder, mass flow rate through the valves, valve dynamics, gas pulsation inside the mufflers and refrigerant thermodynamic properties. Several parameters are calculated during the compressor cycle, such as instantaneous pressure throughout the compressor, mass flow rate, valve dynamics, energy and mass losses, refrigerating capacity, etc.

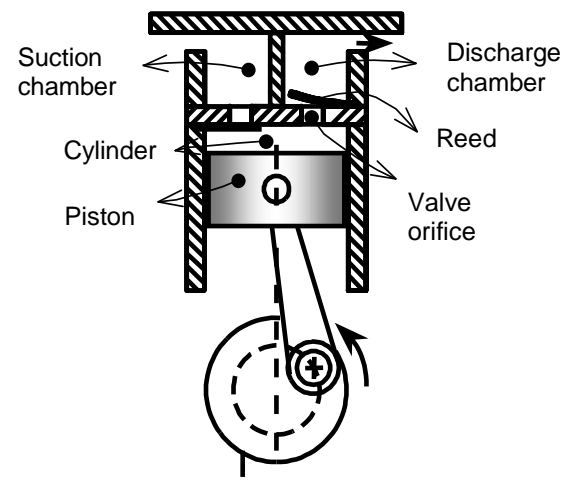

(a)

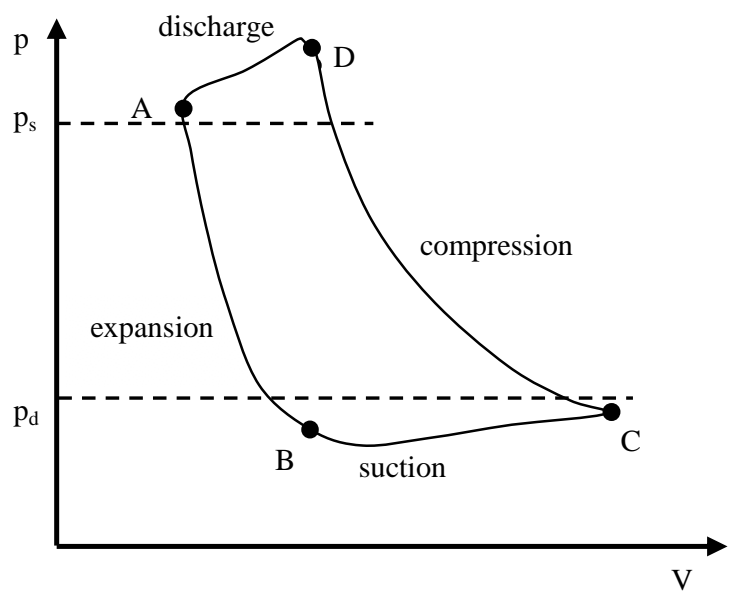

(b)

Figure 1. Schematic of a reciprocating compressor and its indicator diagram.

The transient equations associated with the compressor simulation code are solved via a fourth order Runge-Kutta method. Thermodynamic properties can be evaluated using the perfect gas hypothesis or through a program link to the REFPROP database (Gallanger et al., 1993).

Valve displacement is modeled by a single degree of freedom mass-spring model, in which the natural frequency and damping coefficient have to be specified for the valves. Valve stiction can be obtained from the analytical model proposed by Khalifa and Liu (1998).

The resultant force acting on the reed and the mass flow rate through the valve are obtained with reference to effective force area $A_{e f}$ and effective flow area $A_{e e}$, respectively. From the pressure difference across the valve, $\Delta p_{v}, A_{e f}$ is determined from $A_{e f}=F / \Delta p_{v}$. The effective force area can be understood as a parameter related to how efficiently the pressure difference $\Delta \mathrm{p}_{\mathrm{v}}$ opens the valve. On the other hand, for the same pressure drop, $A_{\mathrm{ee}}$ expresses the ratio between the actual mass flow rate through the valve and that given by an isentropic flow condition.

If specified, gas pulsation in mufflers can be modeled following a methodology developed by Deschamps et al. (2002). The 
thermodynamic process for the gas inside the cylinder can be evaluated either through a polytropic model or by the first law of thermodynamics. More details on the compressor simulation program can be obtained in Fagotti et al. (1994).

\section{Differential Formulation}

For the Reynolds averaged flow equations (RANS), the value of a computed variable represents an ensemble average over many engine cycles at a specified spatial location. In general, twoequation closures, such as the $\mathrm{k}-\varepsilon$ model, have been used to model turbulent transport. The shortcomings of RANS models have been widely documented and a discussion of issues related to reciprocating engines is given in El Tahry and Haworth (1992).

Another approach to accounting for turbulent transport is largeeddy simulation (LES). In LES the governing equations are spatially filtered rather than ensemble averaged. Explicit account is taken of flow structures larger than the filter width, which is in the order of the mesh spacing, while the influence of unresolved scales is modeled using a sub-grid scale (SGS) model. Since statistics of small-scale turbulence are expected to be more universal than those of the large scales turbulence, LES offers the promise of wider generality and reduced modeling uncertainty.

LES is particularly appealing for application to reciprocating engines, given its inherent transient operation, despite its rather undeveloped condition for modeling complex engineering flows, as in the case of reciprocating engines, where moving piston and valves are present. In contrast, in RANS turbulence models the formulation and calibration have traditionally been carried out for statistically stationary flows with simple geometric configurations and, therefore, it is uncertain whether such closures will hold for complex three-dimensional transient flows that characterize reciprocating engines. In addition to this, the grid refinement commonly used for RANS simulations in IC engines is comparable to that required in LES for resolving a significant range of flow structure scales. Given the difficulties associated with RANS models, LES might actually be simpler, less computational expensive and more accurate.

LES is composed of four steps: i) a filtering operation that expresses a generic property $\mathrm{F}(\mathbf{x}, \mathrm{t})$ as the sum of a filtered, or resolved, component, $\mathrm{f}(\mathbf{x}, \mathrm{t})$, and a residual, or sub-grid scale (SGS), component, f'(x, t), where $\mathbf{x}$ and $t$ are the space and time coordinates; ii) the application of a filtering process over the appropriate transport equations, resulting a residual stress tensor for the momentum equation and residual fluxes for scalar transport equations; iii) closure by modeling the residual stress tensor and residual fluxes; iv) the closed filtered equations for $f(x, t)$ are numerically solved for large scale motions.

The contribution of unresolved (sub-grid scale) motions to the resolved scales can be estimated with the concept of an eddy viscosity, $\mu_{t}$, similarly to that used for the Reynolds stress tensor in standard RANS turbulence models. One such a model was proposed by Smagorinsky (1963) and expresses the eddy viscosity as:

$$
\mu_{\mathrm{t}}=\rho\left(\mathrm{C}_{\mathrm{s}} \ell\right)^{2} \sqrt{2 \mathrm{~S}_{\mathrm{ij}} \mathrm{S}_{\mathrm{ij}}}
$$

where $S_{i j}$ is the rate of strain tensor and $\ell$ is a filter width, usually taken as proportional to the local grid spacing, $\Delta$. According to Deardoff (1970), for non-uniform grids $\ell$ can be evaluated as the geometric average of the grid spacing, ie $\ell=(\Delta \mathrm{x} \Delta \mathrm{y} \Delta \mathrm{z})^{1 / 3}$.

The coefficient $\mathrm{C}_{\mathrm{s}}$ is referred to as the Smagorinsky constant and was estimated by Lilly (1967) as being equal to 0.18 for homogeneous and isotropic turbulence. Calibration with respect to benchmark turbulent flows has led modelers to adopt different values for $\mathrm{C}_{\mathrm{s}}$. For homogeneous isotropic decaying turbulence, a value of $\mathrm{C}_{\mathrm{s}}=0.17$ is found to be in good agreement with the experimental data, whereas for planar channel flow, a value of $\mathrm{C}_{\mathrm{s}}=$ 0.1 yields the best agreement. Here $\mathrm{C}_{\mathrm{s}}$ is assumed to be 0.17 .

An important hypothesis in this study is that density fluctuations are negligible when compared to velocity fluctuations. According to Morkovin (1961), this assumption can be justified for non-reacting subsonic flow condition, which is verified in the present situation. Hence, the density variation effect on the flow is accounted for through its spatial and temporal variations.

LES certainly implies a transient three-dimensional simulation. Despite some works in the literature dealing with two-dimensional LES (Iliescu et al., 2003), it should be said this is not really a rigorous approach. However, at this stage of the research it was decided to adopt an axis-symmetric flow condition for additional savings in the computational processing time. To some extent, one could regard such a modeling like an algebraic eddy-viscosity model.

The positioning of the valve in the cylinder head is an important aspect of the compressor design. Although the axis-symmetric geometry for the discharge valve may seem quite idealized, it is a layout found in refrigeration compressors.

By writing residual stresses through the concept of eddy viscosity, the conservation equations for mass, momentum and energy can be written as follows:

a) Mass conservation

$$
\frac{\partial \rho}{\partial \mathrm{t}}+\frac{\partial \rho \mathrm{u}}{\partial \mathrm{x}}+\frac{1}{\mathrm{r}} \frac{\partial \rho \mathrm{rv}}{\partial \mathrm{r}}=0
$$

b) Momentum conservation in the axial (x) direction

$$
\begin{aligned}
\frac{\partial \rho \mathrm{u}}{\partial \mathrm{t}}+\frac{\partial \rho \mathrm{uu}}{\partial \mathrm{x}} & +\frac{1}{\mathrm{r}} \frac{\partial \rho \mathrm{rvu}}{\partial \mathrm{r}}=-\frac{\partial \mathrm{p}}{\partial \mathrm{x}}+\frac{\partial}{\partial \mathrm{x}}\left(\mu_{\text {eff }} \frac{\partial \mathrm{u}}{\partial \mathrm{x}}\right)+\frac{1}{\mathrm{r}} \frac{\partial}{\partial \mathrm{r}}\left(\mathrm{r} \mu_{\text {eff }} \frac{\partial \mathrm{u}}{\partial \mathrm{r}}\right) \\
& +\frac{\partial}{\partial \mathrm{x}}\left(\mu_{\text {eff }} \frac{\partial \mathrm{u}}{\partial \mathrm{x}}\right)+\frac{1}{\mathrm{r}} \frac{\partial}{\partial \mathrm{r}}\left(\mathrm{r} \mu_{\text {eff }} \frac{\partial \mathrm{v}}{\partial \mathrm{x}}\right)-\frac{2}{3} \frac{\partial}{\partial \mathrm{x}}\left(\mu_{\text {eff }} \vec{\nabla} \cdot \overrightarrow{\mathrm{v}}\right)
\end{aligned}
$$

c) Momentum conservation in the radial (r) direction

$$
\begin{array}{r}
\frac{\partial \rho \mathrm{v}}{\partial \mathrm{t}}+\frac{\partial \rho \mathrm{uv}}{\partial \mathrm{x}}+\frac{1}{\mathrm{r}} \frac{\partial \rho \mathrm{rvv}}{\partial \mathrm{r}}=-\frac{\partial \mathrm{p}}{\partial \mathrm{r}}+\frac{\partial}{\partial \mathrm{x}}\left(\mu_{\text {eff }} \frac{\partial \mathrm{v}}{\partial \mathrm{x}}\right)+\frac{1}{\mathrm{r}} \frac{\partial}{\partial \mathrm{r}}\left(\mathrm{r} \mu_{\text {eff }} \frac{\partial \mathrm{v}}{\partial \mathrm{r}}\right) \\
-\frac{\mu_{\text {eff }} \mathrm{v}}{\mathrm{r}^{2}}+\frac{\partial}{\partial \mathrm{x}}\left(\mu_{\text {eff }} \frac{\partial \mathrm{u}}{\partial \mathrm{r}}\right)+\frac{1}{\mathrm{r}} \frac{\partial}{\partial \mathrm{r}}\left(\mathrm{r} \mu_{\text {eff }} \frac{\partial \mathrm{v}}{\partial \mathrm{r}}\right)-\frac{2}{3} \frac{\partial}{\partial \mathrm{r}}\left(\mu_{\text {eff }} \vec{\nabla} \cdot \overrightarrow{\mathrm{v}}\right)
\end{array}
$$

where $\mu_{\text {eff }}$ is the effective viscosity, comprised by the molecular viscosity, $\mu$, and the eddy viscosity, $\mu_{\mathrm{t}}$, ie $\mu_{\mathrm{eff}}=\mu+\mu_{\mathrm{t}}$.

d) Energy conservation

$$
\begin{aligned}
\frac{\partial \rho \mathrm{T}}{\partial \mathrm{t}}+\frac{\partial \rho \mathrm{T} T}{\partial \mathrm{x}}+\frac{1}{\mathrm{r}} \frac{\partial \mathrm{r} \rho \mathrm{vT}}{\partial \mathrm{r}}= & -\mathrm{p} \vec{\nabla} \cdot \overrightarrow{\mathrm{V}}+\frac{\partial}{\partial \mathrm{x}}\left[\left(\frac{\mathrm{k}}{\mathrm{c}_{\mathrm{v}}}+\frac{\mathrm{c}_{\mathrm{p}}}{\mathrm{Pr}_{\mathrm{t}}} \frac{\mu_{\mathrm{t}}}{\mathrm{c}_{\mathrm{v}}}\right) \frac{\partial \mathrm{T}}{\partial \mathrm{x}}\right] \\
& +\frac{1}{\mathrm{r}} \frac{\partial}{\partial \mathrm{r}}\left[\mathrm{r}\left(\frac{\mathrm{k}}{\mathrm{c}_{\mathrm{v}}}+\frac{\mathrm{c}_{\mathrm{p}}}{\operatorname{Pr}_{\mathrm{t}}} \frac{\mu_{\mathrm{t}}}{\mathrm{c}_{\mathrm{v}}}\right) \frac{\partial \mathrm{T}}{\partial \mathrm{r}}\right]
\end{aligned}
$$

where $\mathrm{k}$ is the fluid thermal conductivity and $\operatorname{Pr}_{\mathrm{t}}$ is the turbulent Prandtl number, considered here to be 0.9 .

For simplicity, the perfect gas hypothesis was adopted in the state equation required to complete the equation system. 


\section{Valve Dynamics}

Reeds are usually made of stainless steel and their dynamics can be expressed in a simplified way, using a one degree of freedom model as follows:

$$
\mathrm{m} \ddot{\delta}_{1}+\mathrm{C} \dot{\delta}_{1}+\mathrm{K} \delta_{1}=\mathrm{F}-\mathrm{F}_{0}
$$

where $\mathrm{F}_{0}$ is a pre-load force acting on the reed and $\mathrm{F}$ is the force resulting from the pressure distribution on the reed surface. The valve stiffness and damping coefficients, $\mathrm{K}$ and $\mathrm{C}$, respectively, as well as the valve mass, $\mathrm{m}$, are determined experimentally. As illustrated in Fig. 2, in this study the reed is considered to be parallel to the valve seat.

In order to solve Eq. (6) for the valve lift $\delta_{1}$, a fourth order Runge-Kutta methodology was adopted. The force $\mathrm{F}$ is evaluated from the pressure field created by the flow through the valve:

$$
\mathrm{F}=\int_{0}^{\mathrm{D} / 2} \mathrm{p} 2 \pi \mathrm{rdr}
$$

It should be mentioned that for the discharge valve, the valve lift was limited to $0.9 \mathrm{~mm}$ and a booster was set to act when the valve displacement was $0.3 \mathrm{~mm}$. Since the flow through the suction valve is not solved, the force $\mathrm{F}$ on the suction reed has to be obtained via the effective force area, $\mathrm{A}_{\mathrm{ef}}$.

\section{Numerical Methodology}

\section{Moving Coordinate System}

A key issue in solving the governing equations is the methodology chosen to handle the physical domain that expands and contracts, since both the piston and the valves are in motion. Here, following the practice adopted and fully described in Matos et al. (1999), a moving coordinate system was employed. The main feature of this coordinate system is that it transforms the physical domain shown in Fig. 2 into a computational domain that remains unchanged regardless any surface motion.

\section{Solution Procedure}

A finite volume methodology was employed to integrate the partial differential equations governing the flow, with a fully implicit approximation for transient terms. Staggered control volumes were adopted for velocity components. Interpolation of unknown quantities at the control volume faces was obtained using the QUICK interpolation scheme (Hayase et al., 1992). A segregate approach was employed to solve the equations and the coupling between pressure and velocity was handled through the SIMPLEC algorithm.

The system of algebraic equations that result from the integration of the governing differential equations over each control volume are solved using the Tridiagonal Matrix Algorithm (TDMA).

The solution domain was discretized with a computational grid with 110x90 (x, r) volumes, as illustrated in Fig. 2. Grid refinement was specifically adopted in regions where high gradients are expected to occur in the flow field. Of great help was some evidence of the discretization needed for the flow analysis in the discharge valve as made available by Salinas-Casanova et al. (1999). For instance, Fig. 3 shows a typical result emerging from the assessment of truncation error in the numerical results for pressure distribution on the reed surface, considering a fixed reed lift, $\mathrm{s} / \mathrm{d}=0.05$, and a
Reynolds number, $\operatorname{Re}=25,000$, based on the valve orifice diameter d. The results yielded by three grid levels (70x80, 140x160 and $280 \times 320$, axial $x$ radial) show that the less refined grid returns a small truncation error and, therefore, a similar grid was adopted for the compressor simulation. Further details on this issue and on the computational domain dimensions can be found in Matos (2002).

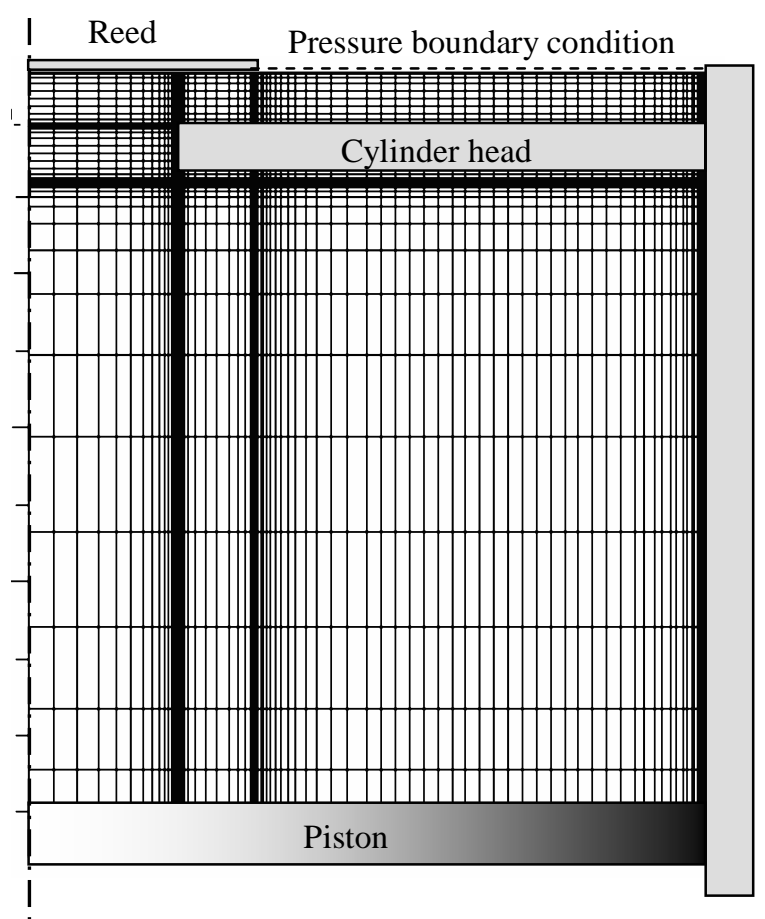

Figure 2. Physical domain and computational grid.

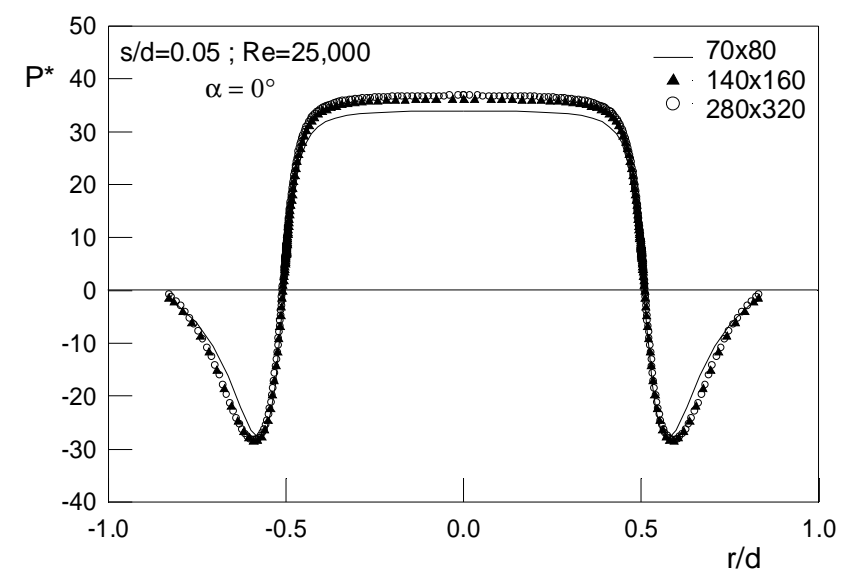

Figure 3: Sensitivity of results for pressure distribution to mesh refinement.

The iterative procedure evaluates flow properties for each time step until convergence is reached, which is ascertained by examining whether the compressor operation conditions are cyclically repeated. A time step corresponding to $10^{-2} \mathrm{rad}$ was employed for the integral approach, whereas a time step of $10^{-3} \mathrm{rad}$ was needed for the differential formulation to guarantee the numerical convergence. Further refinement in the time step did not produce any modification in the numerical solution. However, it should be mentioned that a smaller time step, $10^{-4} \mathrm{rad}$, was required when the discharge valve was open due to steep flow transients. By 
using such time steps, 4 cycles were required to establish a periodic condition, taking approximately 39 hours on a computer with a single Pentium IV $2 \mathrm{GHz}$ processor.

The differential equation for the valve dynamics, Eq. (6), was solved by considering the force $\mathrm{F}$ to be constant during each time step. As already indicated, in the case of the discharge valve $\mathrm{F}$ is obtained from the pressure distribution caused by the flow on the reed surface. However, for the suction valve $\mathrm{F}$ must be calculated from effective force area data.

The coupling between integral and differential formulations needs to be addressed. The compressor simulation starts at the bottom dead center (BDC) with the integral formulation, as represented in the illustrative diagram shown in Fig. 4. The cylinder pressure is the parameter chosen as the reference for change from one formulation to the other. In this way, during the compression stroke the integral formulation is changed to the differential formulation when the pressure reaches a value set at $12 \mathrm{bar}$. At this point, values for pressure, $p$, temperature, $T$, and density, $\rho$, are directly transferred and used as initial fields for the differential formulation. Other quantities given are the instantaneous piston position and cylinder volume $\mathrm{V}$, both related to the crankshaft angle wt [ rad], where $\mathrm{w}=2 \pi \mathrm{f}$ and $\mathrm{f}=60 \mathrm{~Hz}$. No information is available for the velocity field and its initial value is set to zero. For the eddy viscosity, $\mu_{\mathrm{t}}$, an estimate is obtained from the numerical solution given in Matos (2002). After the gas is pushed out from the cylinder, the discharge valve is closed and the compressor is under the expansion stroke. The procedure is changed back to the integral formulation when the pressure reaches 8.5 bar. Besides allowing the solution of the flow through the discharge valve, the choice of this crankshaft angle range (12 to 8.5 bar) for the differential formulation has also some advantages. Firstly, at 12 bar the piston is very near the top dead center and the in-cylinder flow field is almost zero due the proximity between the piston and the cylinder head, which may be as close as $30 \mu \mathrm{m}$. This is an adequate position to change from the integral to the differential formulation. On the other hand, at 8.5 bar, the in-cylinder flow induced by the discharge valve opening is sufficiently damped so that mean values necessary to the integral formulation are not far from the flow field condition.

Since flow quantities are available for each control volume of the domain, an average value is required for pressure, temperature and density.

\section{Boundary Conditions}

At the solid walls all velocity components were taken to be zero, except at the surfaces of the reed and piston where $u$ represents their corresponding velocity values; i.e. $\dot{\delta}_{1}$ and $\dot{\delta}_{3}$, respectively. At the valve axis $(\mathrm{r}=0)$ the axis-symmetry conditions were imposed as $\mathrm{v}=$ $\partial \mathrm{u} / \partial \mathrm{r}=0$. At the valve outlet, a boundary condition for pressure, equal to 14.8 bar, was prescribed. To obtain the axial velocity from the specified pressure value, a control volume half that of the control volumes adjacent to the boundary was used to integrate the axial momentum equation. By doing so, the velocity could be expressed in terms of the pressure gradient and the neighboring velocity components. More details on this technique can be obtained from Versteeg and Malalasekera (1995). Because the axial velocity obtained from this equation satisfied the momentum conservation but not the mass conservation, such an estimate had to be corrected via the SIMPLEC algorithm, in the same way as in the solution domain. For the velocity component parallel to the outlet boundary, the prescribed condition was $\partial v / \partial x=0$.

Concerning boundary conditions for the energy equation, a value of $90{ }^{\circ} \mathrm{C}$ was prescribed for the wall temperature. For the discharge, a local parabolic flow condition was used when the gas is leaving the domain. If the flow is entering the domain, the temperature at the boundary is considered to be that in the discharge chamber.

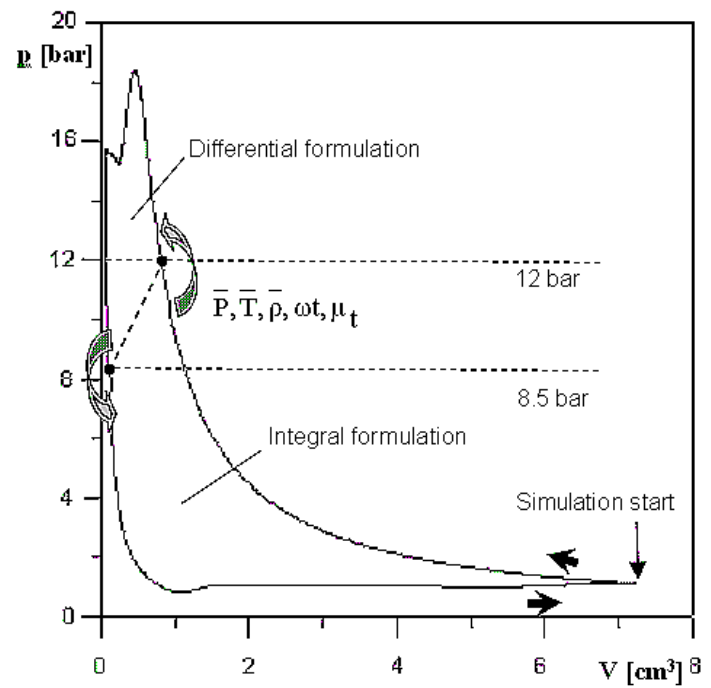

Figure 4. Schematic of interaction between integral and differential formulations.

\section{Results}

The results given next were prepared for four crankshaft positions during the opening of the discharge valve: $w t=2.67$ (a); 2.80 (b); 2.99 (c) and 3.13 (d) rad. Such positions are identified in Fig. 5, which shows the results for pressure at the cylinder wall and valve displacement, according to the crankshaft angle. The precise location of the pressure monitoring at the cylinder wall is indicated in Fig. 6a. Each point can be associated with the following events during the compressor stroke: (a) discharge valve is opening and the cylinder pressure is near its highest value; (b) discharge valve is still opening and the pressure has dropped; (c) valve is closing and the pressure has increased; (d) valve is returning to the valve seat and pressure is decreasing.

The pressure increase to a level above that of the discharge chamber is linked to distinct aspects: i) stiction force between reed and valve seat caused by a film of oil; ii) valve inertia; iii) increase in stiffness and natural frequency due to the presence of a booster; iv) flow restriction through the valve. The first two effects are present when the valve is closed.

By examining Fig. 5, it is possible to identify the opening of the valve when the cylinder pressure reached 15 bar. After the valve is opened, the pressure keeps rising up to point (a) due to the flow restriction brought about by a small valve lift.

The discharge valve keeps opening and, when the lift is great enough, the cylinder pressure starts to drop down to point (b). From this crankshaft position the pressure is seen to increase again, reaching a new local maximum condition, point (c). This occurs for two reasons: i) an increase in the viscous friction of the flow, due to the small clearance between the piston and the cylinder head; ii) closing motion of the valve, restricting the flow passage area. The viscous friction effect in the flow along the cylinder clearance can be observed from the results for the isobars, shown in Fig. 6. As can be seen in Fig. 6c, there is a pressure drop of approximately 1 bar between the cylinder wall and the valve orifice. The same level of pressure drop is not observed for points (a) and (b).

For the compressor model here considered, the top dead center (TDC) is reached at a crankshaft angle of $3.10 \mathrm{rad}$. Since point (d) represents an angle of $3.13 \mathrm{rad}$, here the piston has already started 
the expansion stroke, resulting in an abrupt pressure drop in the cylinder that acts to close the discharge valve.

Results for dimensionless streamlines $\psi^{*}(=\psi / \dot{\mathrm{m}})$ in Fig. 7 show the presence of recirculating regions on the valve seat and inside the valve orifice, during the discharge. This phenomenon has an adverse effect on the valve efficiency since it restricts the flow passage, thus bringing about a further increase in the cylinder pressure. When the valve is almost fully open, the discharge jet at the valve outlet induces a recirculating flow region against the vertical wall present in the right side of the solution domain.

The pressure distribution on the valve surface is a key factor in the valve design since it dictates the resultant force responsible for the reed motion. The presence of a recirculating region on the valve seat may return low pressure levels on the reed surface. This may prevent the perfect valve operating conditions since it reduces the force acting on the reed surface. In view of this, the correct prediction of eventual recirculating regions is a key aspect in the compressor simulation.

The ratio between the diameters of the reed and valve orifices is approximately 1.5 for the compressor being simulated. The diffuser region, situated between the reed and the valve seat, does not have sufficient length to allow either a complete flow development or pressure recovery.

As the valve lift is increased, and the flow inertia becomes stronger, the separation region is increased and the recirculating zone moves into the valve outlet. In such situations, the pressure at the valve outlet may even not reach the discharge pressure condition, giving rise to flow entrainment, as shown in Fig. 7b.

Figure 8 shows the results for vector velocities in zoomed regions of the flow, all of them identified in Fig. 7. Naturally, many of the flow features examined through results for the streamlines can also be observed from these results, such as the flow entrainment in Fig 8a. On the other hand, Fig. 8b shows that the recirculating flow region on the valve seat virtually vanishes at $\mathrm{wt}=2.99 \mathrm{rad}$, point (c), although the valve lift is approximately the same of that at $\mathrm{wt}=$ $2.67 \mathrm{rad}$. There are two reasons for this difference. Firstly, the pressure drop through the valve has significantly decreased when the compressor reaches $\mathrm{wt}=2.67 \mathrm{rad}$. This reduces the mass flow rate through the valve and, as consequence, the same happens with the inertia effects that act on the flow separation. The other aspect, which is less obvious, is that the reed closing motion induces a pressure field that inhibits any flow separation.

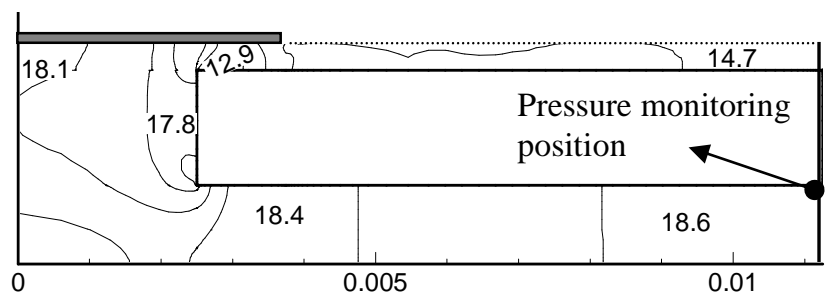

(a) $\mathrm{wt}=2.67 \mathrm{rad}$

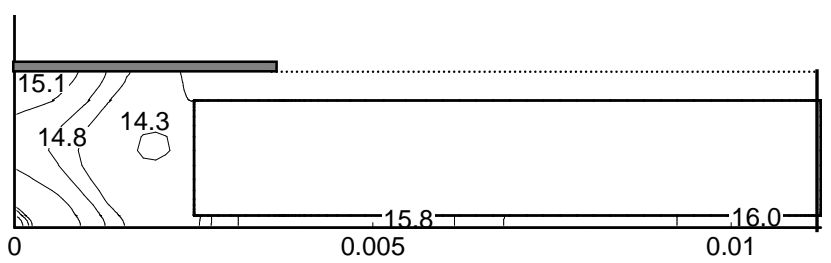

(c) $\mathrm{wt}=2.99 \mathrm{rad}$
An important aspect captured by the simulation, and difficult to detect from streamlines, is the backflow seen between the reed and the seat during the reed closing motion, as can be seen in Fig. 8c for the crankshaft angle wt $=3.13 \mathrm{rad}$. This is a very undesirable feature related to the valve dynamics that reduces the volumetric efficiency of the compressor. Therefore, in the design of an efficient valve system the dynamics must be tuned so as to minimize this aspect.

At wt $=3.13 \mathrm{rad}$ the piston has already passed through TDC and the in-cylinder flow is seen to occur from the valve orifice to the cylinder wall, owing to the downward piston motion (Fig. 8d).

The pressure field represented in Fig 6d is in line with the valve backflow and also with the flow in the cylinder clearance. Although the pressure difference between the valve outlet and the valve orifice is not very significant, since the backflow rate is not high, the pressure gradient along the cylinder head clearly indicates the flow induced by the piston motion.

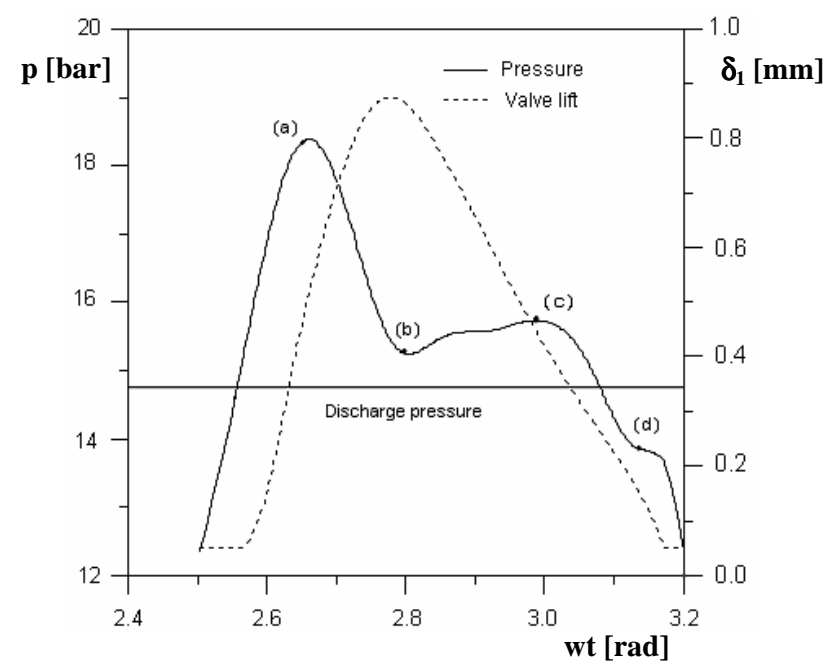

Figure 5. Cylinder pressure, valve lift and monitoring positions during the discharge process.

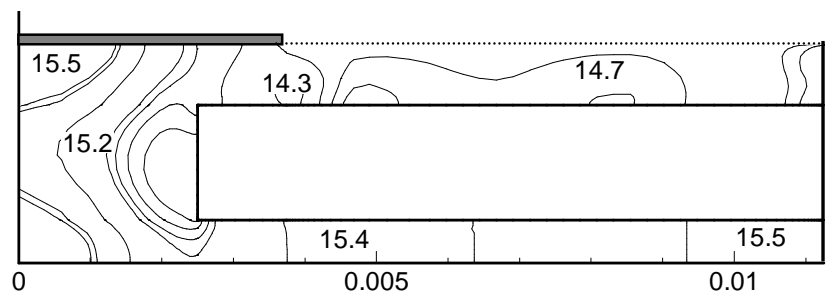

(b) $\mathrm{wt}=2.80 \mathrm{rad}$

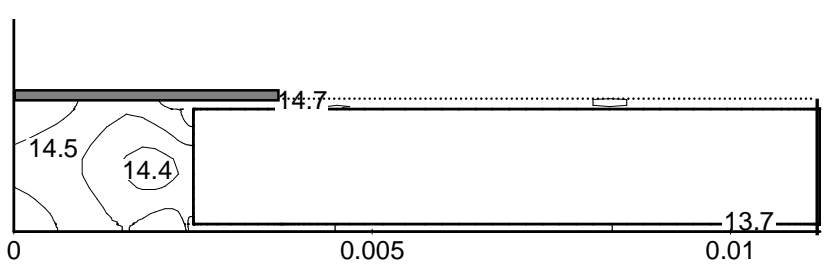

(d) $\mathrm{wt}=3.13 \mathrm{rad}$

Figure 6. Isobars for the flow through the discharge valve. 


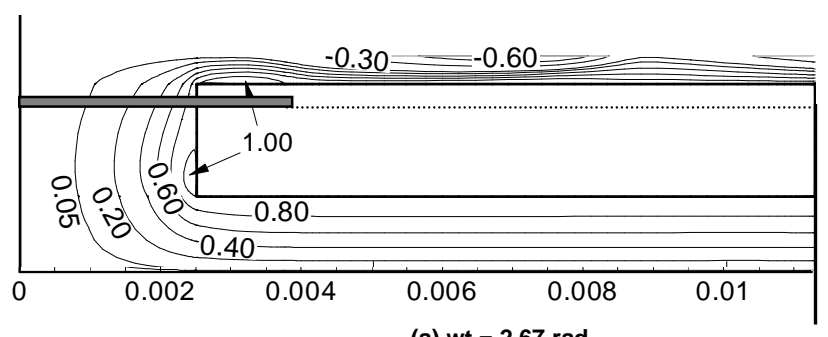

(a) $\mathrm{wt}=2.67 \mathrm{rad}$

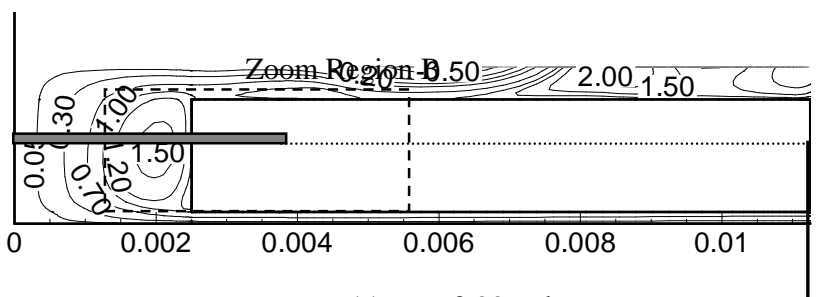

(c) $\mathrm{wt}=2.99 \mathrm{rad}$

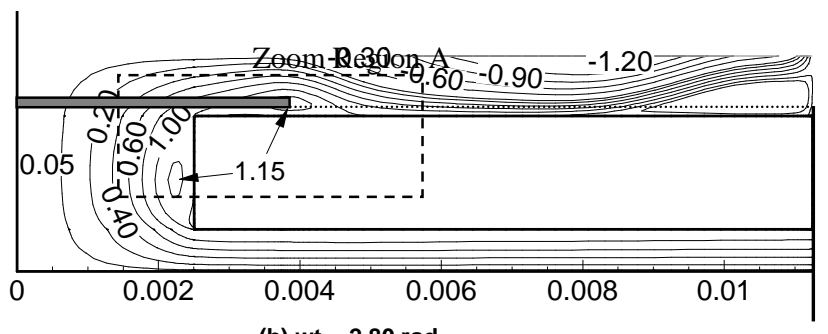

(b) $\mathrm{wt}=2.80 \mathrm{rad}$

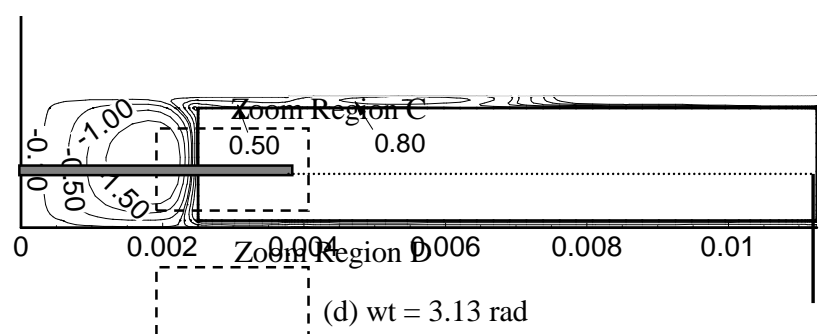

(d) $\mathrm{wt}=3.13 \mathrm{rad}$

Figure 7. Dimensionless streamlines.

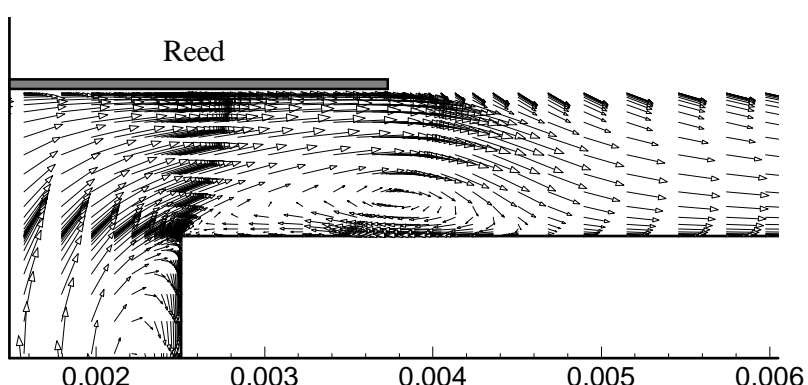

(a) Zoom region $\mathrm{A}$

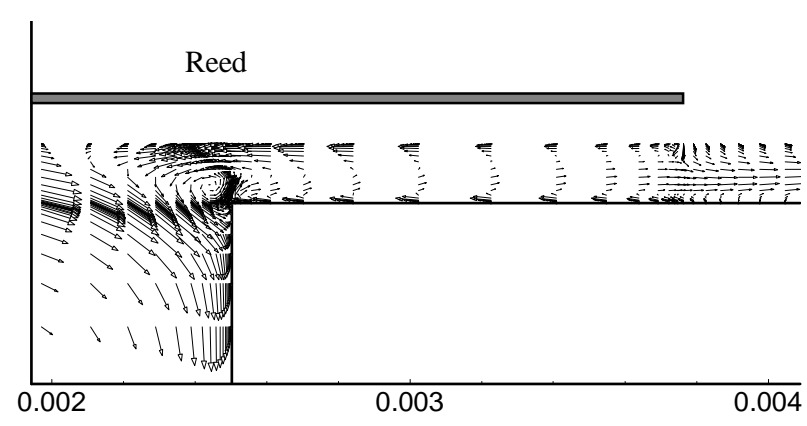

(c) Zoom region $\mathrm{C}$

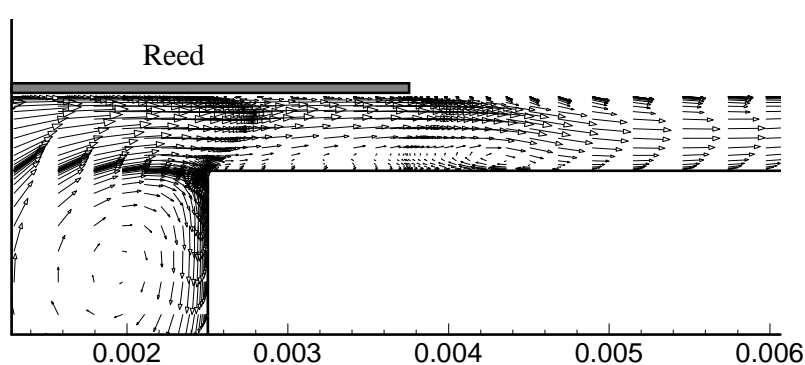

(b) Zoom region $B$

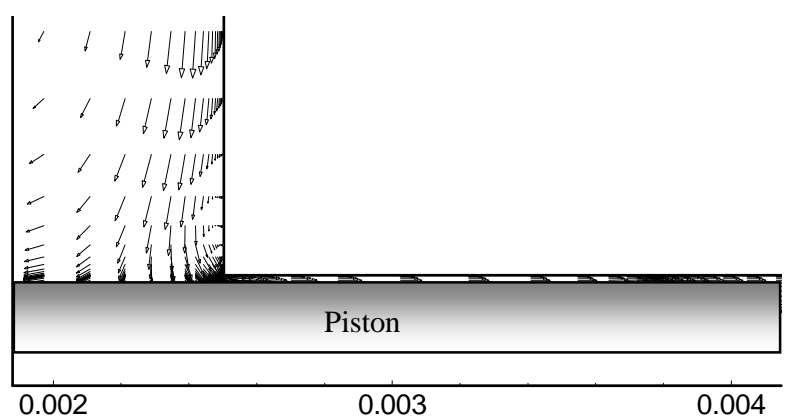

(d) Zoom region $\mathrm{D}$

Figure 8. Flow velocity vectors during the discharge process.

Figure 9 shows results for the ratio between effective viscosity, $\mu_{\text {eff }}=\mu_{\mathrm{t}}+\mu$, and molecular viscosity, $\mu$, at two crankshaft positions, $\mathrm{wt}=2.80$ and $2.99 \mathrm{rad}$, during the compressor discharge process. The eddy viscosity levels predicted by the Smagorinsky model, Eq. (1), are directly related to the rate of strain $S_{i j}$. Accordingly, high levels of $\mu_{\text {eff }} / \mu$ occur at wt $=2.80$, due to the flow strain in the valve orifice and in the cylinder head. As the piston approaches the top dead center, the velocity magnitude in the gap between the piston and the cylinder head decreases and, as a consequence, the same occurs with the levels of $\mu_{\text {eff }} / \mu$ there. This can be observed by comparing results of $\mu_{\mathrm{eff}} / \mu$ for wt $=2.80$ and $2.99 \mathrm{rad}$. 


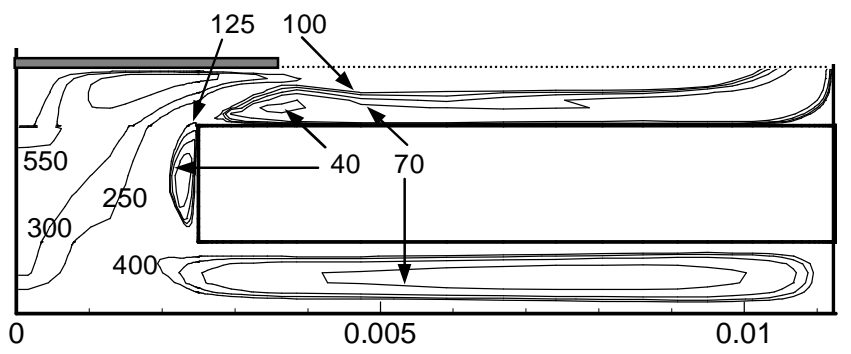

(a) $\mathrm{wt}=2.80 \mathrm{rad}$

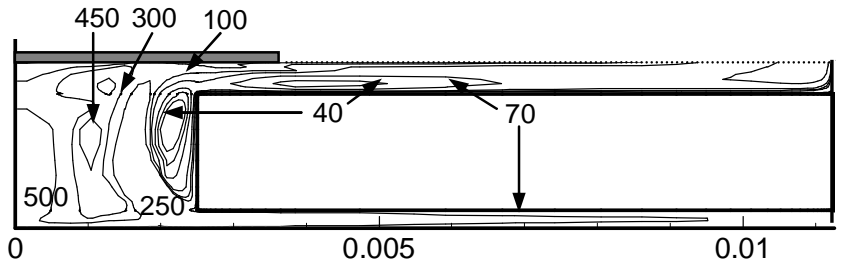

(b) $\mathrm{wt}=2.99 \mathrm{rad}$

Figure 9. Isolines for ratio between effective viscosity and molecular viscosity.

\section{Conclusions}

A methodology has been proposed to simulate reciprocating compressors, with particular attention given to the discharge valve dynamics. In order to reduce the computational time, the solution procedure combines integral and differential formulations. The compressible turbulent flow through the discharge valve was predicted via large-eddy simulation (LES), with the Smagorinsky model for the sub-grid scale motions. Results were seen to capture several important phenomena that occur in the discharge process, such as pressure over shooting in the cylinder and backflow through the discharge valve. Nevertheless, the methodology still requires to be fully validated, with reference to experimental data, before a more conclusive analysis can be provided. In addition to that, future developments will be concentrated on extending the simulation to a three-dimensional geometry and implementing alternative sub-grid scale models.

\section{Acknowledgments}

This work forms part of a technical-scientific cooperation program between Federal University of Santa Catarina and EMBRACO. Support from the Brazilian Research Council, CNPq, is also acknowledged.

\section{References}

Deardorff, J.M., 1970, “A Numerical Study of Three-Dimensional Turbulent Channel Flow at Large Reynolds Number", J. Fluid Mechanics, Vol. 41, pp. 453-480.

Deschamps, C.J., Prata, A.T. and Ferreira, R.T.S., 1996, "Turbulent Flow through Reed Type Valves of Reciprocating Compressors", Proc. ASME International Congress and Exposition - Symposium on the Analysis and Applications of Heat Pump \& Refrigeration Systems, Texas, USA, pp. 151-161.

Deschamps, Possamai, F.C. and Pereira, E.L.L, 2002, "Numerical Simulation of Pulasting Flow in Suction Mufflers", Proc. Compressor Engineering Conference at Purdue (CD-ROM), Indiana, USA, 8p.

El Tahry, S.H., Haworth D.C., 1992, "Directions in Turbulence Modeling for In-Cylinder Fows in Reciprocating IC Engines". AIAA J Prop and Power, Vol. 8, pp.1040-1048.

Fagotti, F., Todescat, M.L., Ferreira, R.T.S., Prata, A.T., 1994, "Heat Transfer Modeling in a Reciprocating Compressor", Proc. Compressor Engineering Conference at Purdue, Indiana, USA, pp. 605-610.

Gallanger, J., McLinden, M., Morrison, G. and Huber, M., 1993, "NIST Thermodynamic Properties of Refrigerants and Refrigerants Mixtures Database (REFPROP)", Thermophysics Division, National Institute of Standards and Technology, US Department of Commerce, USA.

Hayase, T., Humphrey, J.A.C., and Greif, R., 1992, "A Consistently Formulated QUICK Scheme for Fast and Stable Convergence Using FiniteVolume Iterative Calculation Procedures", J. Comp. Physics, Vol. 98, pp. 108-118.
Iliescu, T, John, V., Layton, W.J., Matthies, G. and Tobiska, L., 2003, "A Numerical Study of a Class of LES Models", Int. J. Computational Fluid Dynamics, Vol. 17, pp. 75-85.

Khalifa, E.H. and Liu, X., 1998, "Analysis of Stiction Effect on the Dynamics of Compressor Suction Valve", Proc. International Compressor Engineering Conference at Purdue, USA, Vol. I, pp. 87-92.

Lilly, D.K., 1967, "The Representation of Small-Scale Turbulence in Numerical Simulation Experiments”. Proc. of the IBM Scientific Computing Symposium on Environmental Sciences, Goldstine, H. H. ed., IBM Form No. 320-1951, pp. 195-210.

Lopes, M.N. and Prata, A.T., 1997, "Dynamic Behavior of Plate Type Valves in Periodic Flows" (in portuguese), COB 1138, Proc. XIV Brazilian Congress of Mechanical Engineering (CD-ROM), Bauru, Brazil.

MacLaren, J.F.T., Tramscheck, A.B., Sanjines, A., Pastrana, O.F., 1975, "A Comparison of Numerical Solutions of Unsteady Flow Equations applied to reciprocating Compressor Systems”, J. Mechanical Engineering Science, Vol. 17, pp. 271-279.

Matos, F.F.S., Prata, A.T., Deschamps, C.J., 1999, "Numerical Analysis of the Dynamic Behaviour of Plate Valves in Reciprocating Compressors", Proc. International Conference on Compressor and Their Systems, London, UK, pp. 453-462.

Matos, F.F.S., Deschamps, C.J., Prata, A.T., 2002, "Numerical Simulation of Turbulent Flow in Reciprocating Compressors" (in Portuguese). Proc. of the 2002 Spring School on Transition and Turbulence (CD-ROM). Florianópolis, Brazil, $10 \mathrm{p}$.

Matos, F.F.S., 2002, "Numerical Analysis of the Dynamic Behavior of Compressor Reed type Valves" (in Portuguese). Ph.D. Thesis, Departamento de Engenharia Mecânica, Universidade Federal de Santa Catarina. Florianópolis, SC, Brazil.

Matos, F.F.S., Prata, A.T., Deschamps, C.J., 2002, "Numerical Simulation of the Dynamics of Reed Type Valves", Proc. Compressor Engineering Conference at Purdue (CD-ROM), Indiana, USA, 8 p.

Morkovin, M.V., 1961, "Effects of compressibility on turbulent flows". In Mecanique de la Turbulence (ed. A. Favre), Editions du Centre National de la Recherche Scientique, Paris, pp. 367-380.

Pérez-Segarra, C.D., Escanes, F., Oliva, 1994, "Numerical Study of the Termal and Fluid Dynamic Behavior of Reciprocating Compressors", Proc. Compressor Engineering Conference at Purdue, Indiana, USA, pp. 145-150.

Salinas-Casanova, D.A., Deschamps, C.J., Prata, A.T., 1999, "Turbulent

Flow through a Valve with Inclined Reeds". International Conference on Compressor and Their Systems, London, UK, pp. 443-452.

Smagorinsky, J.S., 1963, "General Circulation Experiments with the Primitive Equations: I. The Basic Experiment”. Mon. Weath. Rev., Vol. 91, pp. 99-163.

Soedel, W., 1972, "Introduction to Computer Simulation of Refrigerating Compressors", Purdue University Short Courses, Indiana, USA.

Trella, T.J.; Soedel, W., 1974, "Effect of Valve Port Gas Inertia on Valve Dynamics - Part I: Simulation of Poppet Valve”. Proc. Compressor Engineering Conference at Purdue, pp. 190-197.

Ussyk, M.S., 1984, "Numerical Simulation of Hermetic Reciprocating Compressors" (in Portuguese). M.Sc. Thesis, Departamento de Engenharia Mecânica, Universidade Federal de Santa Catarina. Florianópolis, SC, Brazil.

Versteeg, H.K. and Malalasekera, W., 1995, "An Introduction to Computational Fluid Dynamics”, Longman Scientific \& Technical, England, $257 \mathrm{p}$. 